Public Abstract

First Name:Bo

Middle Name:

Last Name:Wang

Adviser's First Name:Zhihai

Adviser's Last Name:He

Co-Adviser's First Name:

Co-Adviser's Last Name:

Graduation Term:FS 2007

Department:Electrical Engineering

Degree:PhD

\title{
Title:Distributed Resource Allocation and Performance Optimization for Video Communication over Mesh Networks Based on Swarm Intelligence
}

Mesh networking technologies allow a system of communication devices to communicate with each other over a dynamic and self-organizing wired or wireless network from everywhere at anytime. Large-scale mesh communication networks involve a large number of heterogeneous devices, each with different onboard computation speeds, energy supplies, and communication capabilities, communicating over the dynamic and unreliable networks. How to coordinate the resource utilization behaviors of these devices in a large-scale mesh network remains a challenging task. And an effective solution to this type of problems needs to meet the following three requirements: distributed, asynchronous, and non-convex.

In this work, based on swarm intelligence principles, we develop a set of distributed and asynchronous schemes for resource allocation and performance optimization for a wide range of mesh networking-based applications, including PSO with convex mapping, Decentralized PSO, Distributed and Asynchronous PSO and Energy Efficient DAPSO. To successfully apply the swarm intelligence principle in distributed resource allocation and performance optimization in large-scale mesh networks, there are three important issues that need to be carefully investigated. First, existing PSO schemes are not able to efficiently handle constraints, especially constraints in a high-dimensional space. To address this issue, we propose to transform the solution space defined by resource constraints into a convex region in a low-dimensional space. We then merge the convex condition with the swarm intelligence principle to guide the movement of each particle to efficiently search for the optimum solution. Second, distributed optimization requires decomposition of centralized network utility function and resource constraints into local ones. In this work, we propose various methods and approaches for decomposition of network utility function and interwound resource constraints. Third, one of the key challenges in resource allocation and performance optimization is to handle critical / bottleneck links which have very limited resource however are shared by multiple video communication sessions. To address this issue, we propose various schemes to fuse the resource allocation information of neighboring optimization modules, propagate and share the resource allocation results at critical links, use this external information to guide the movements of particles in each local optimization module to efficiently search for the optimum solution.

Our extensive experimental results in distributed resource allocation and performance optimization demonstrate that the proposed schemes work efficiently and robustly. Compared to existing algorithms, including gradient search and Lagrange optimization, the proposed approach have the advantage of faster convergence and the ability to handle generic network utility functions. Compared to centralized performance optimization schemes, the proposed approach significantly reduces communication overhead while achieving similar performance. The distributed algorithms for resource allocation and performance optimization provide analytical insights and important guidelines for practical design of large-scale video mesh networks. 